\title{
Trace map, Cayley transform and LS category of Lie groups
}

\author{
A. Gómez-Tato · E. Macías-Virgós · \\ M. J. Pereira-Sáez
}

\begin{abstract}
The aim of this paper is to use the so-called Cayley transform to compute the LS category of Lie groups and homogeneous spaces by giving explicit categorical open coverings. When applied to $U(n), U(2 n) / S p(n)$ and $U(n) / O(n)$ this method is simpler than those formerly known. We also show that the Cayley transform is related to height functions in Lie groups, allowing to give a local linear model of the set of critical points. As an application we give an explicit covering of $S p(2)$ by categorical open sets. The obstacles to generalize these results to $S p(n)$ are discussed.
\end{abstract}

Keywords LS category · Cayley transform · unitary group · symplectic group · Bott-Morse function · left eigenvalue

Mathematics Subject Classification (2000) 55M30 (Primary) · 22E15, 58E05 (Secondary)

\section{Contents}

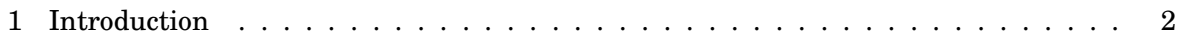

2 The Cayley transform ............................ 3

2.1 Preliminaries ........................ 3

2.2 Generalized Cayley transform . . . . . . . . . . . . . . . . . 3

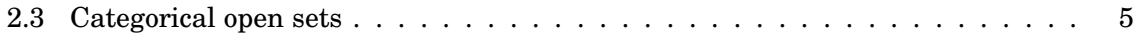

3 Bott-Morse functions on Lie groups . . . . . . . . . . . . . . . . . . . 5

Partially supported by FEDER and Research Project MTM2008-05861 MICINN Spain

A. Gómez-Tato

Institute of Mathematics, Department of Geometry and Topology

University of Santiago de Compostela, 15782- Spain

E-mail: antonio.gomez.tato@usc.es

E. Macías-Virgós

E-mail: quique.macias@usc.es

M. J. Pereira-Sáez

E-mail: mariajose.pereira@usc.es 
3.1 Critical points of a height function . . . . . . . . . . . . 6

3.2 Gradient flow and local model of the critical point set . . . . . . . . . . . 7

4 Applications to LS category . . . . . . . . . . . . . . . . . . . . 9

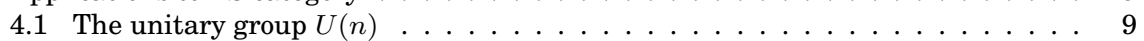

4.2 The symmetric spaces $U(2 n) / S p(n)$ and $U(n) / O(n) \ldots \ldots \ldots \ldots$

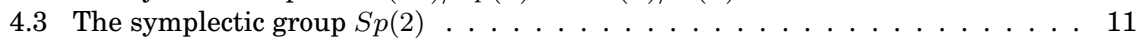

4.4 Left eigenvalues of symplectic matrices $\ldots \ldots \ldots \ldots \ldots \ldots \ldots$

\section{Introduction}

Lusternik-Schnirelmann category is a homotopical invariant that has been widely studied [4,11]. For a topological space $X$, the LS category cat $X$ is defined as the minimum number (minus one) of categorical open sets which are needed to cover $X$ (an open set is categorical when it is contractible in $X$ ).

Unfortunately the LS category is very difficult to compute. For instance, while the result cat $S p(2)=3$ was proven by P. Schweitzer in 1965 [18], it was not until 2002 that cat $S p(3)=5$ appeared in [7], see also [10]. In general, the algebraic techniques involved are highly elaborated. It is then of interest to introduce more elementary methods.

The main idea of this paper is to compute the LS category of some Lie groups and homogeneous spaces of the orthogonal type by means of the so-called Cayley transformation. This will give proofs - which are simpler than the original ones-, of cat $U(n)=n$ (by W. Singhof [19]), cat $U(n) / S p(n)=n$ and cat $U(n) / O(n)=n$ (by M. Mimura and K. Sugata [14]).

Our method is closely related to Morse theory on Lie groups. Classically, the functions that use to be considered are "height" or "distance" as in [5,21,22]. On a matrix Lie group $G$ these functions are, up to a constant, of the form $h_{X}(A)=\Re \operatorname{Tr}(X A)$, the real part of the trace, for some matrix $X$, a fact which allows to explicitly describe the Bott-Morse structure of these functions. We shall prove that the Cayley transform serves to linearize the gradient flow of $h_{X}$ and to give local charts for the set of critical points. These results generalize those of K. Y. Volchenko and A. N. Kozachko ([22], see also [21]).

Let us remember that in a compact manifold the LS category (plus one) is a lower bound for the number of critical points of any smooth function (Morse or not). The reason is that -roughly speaking- for each critical point the gradient flow defines a categorical open set. What is nice in our setting is that this flow is given by the contraction associated to the Cayley map.

Moreover, the Morse interpretation above allows us to give an explicit covering of $S p(2)$ by four categorical open sets, a result that completes the abstract proof by Schweitzer [18].

At the end of the paper we explain how the generalization of our results to the symplectic group $S p(n)$ depends on the computation of the so- 
called left eigenvalues of a quaternionic matrix [25], a topic about which very little is known, out of the case $n=2$.

We hope that the ideas presented here will deserve further attention.

\section{The Cayley transform}

The classical Cayley transform was introduced by A. Cayley in 1846 [3], as a way to express an orthogonal transformation by means of skewsymmetric coordinates. It is given by

$$
c(X)=\frac{I-X}{I+X} .
$$

This map is defined for all matrices having their eigenvalues different from -1 and equals its own inverse, $c^{2}=\mathrm{id}$. It can be thought as a generalization of the stereographic projection.

Its basic properties appear in [17], see also [23].

In order to obtain a categorical covering of the orthogonal Lie groups we shall introduce in the next paragraphs a convenient generalization of the classical Cayley map.

\subsection{Preliminaries}

Let the algebra $\mathbb{K}$ be either $\mathbb{R}$ (reals), $\mathbb{C}$ (complex) or $\mathbb{H}$ (quaternions). We say that the matrix $A \in \mathcal{M}(n, \mathbb{K})$ is orthogonal if $A A^{*}=\mathrm{id}$, where $A^{*}=\bar{A}^{t}$ is the conjugate transpose. Such a matrix can be identified with a (right) $\mathbb{K}$-linear map $\mathbb{K}^{n} \rightarrow \mathbb{K}^{n}$ preserving the product $\langle v, w\rangle=v^{*} w$. Let us denote by $G=O(n, \mathbb{K})$ the Lie group of orthogonal matrices. Depending on $\mathbb{K}$ this group corresponds to the orthogonal group $O(n)$, the unitary group $U(n)$ or the symplectic group $S p(n)$.

Remark 1 The Cayley transform maps a classic orthogonal Lie group like $G=U(n)$ or $S p(n)$ into its Lie algebra $\mathfrak{g}$ of skew-hermitian matrices. In fact, suppose that $A$ is a unitary or symplectic matrix. It can be diagonalized, $A=U D U^{*}$, to a complex diagonal matrix $D=\operatorname{diag}\left(\lambda_{1}, \ldots, \lambda_{n}\right)$ [2]. Then

$$
c(A)=U \operatorname{diag}\left(\pi\left(\lambda_{1}\right), \ldots, \pi\left(\lambda_{n}\right)\right) U^{*},
$$

where $\pi$ is the stereographic projection $\pi: S^{1} \backslash\{-1\} \rightarrow \mathbb{R}$.

\subsection{Generalized Cayley transform}

Let $A \in O(n, \mathbb{K})$ be an orthogonal matrix, where $\mathbb{K}$ is $\mathbb{R}, \mathbb{C}$ or $\mathbb{H}$. 
Definition 1 Let us denote by $\Omega(A) \subset \mathcal{M}(n, \mathbb{K})$ the open set of matrices $X$ such that $A+X$ is invertible. The Cayley transform centered at $A$ is the map

$$
c_{A}: \Omega(A) \rightarrow \Omega\left(A^{*}\right)
$$

given by

$$
c_{A}(X)=\left(I-A^{*} X\right)(A+X)^{-1} .
$$

The classical Cayley map corresponds to $A=I$. As we shall see in the next Proposition, the application $c_{A}$ is well defined and it is invertible, with $c_{A}^{-1}=c_{A^{*}}$.

Proposition 1 If $X \in \Omega(A)$ then

1. $c_{A}(X)=(A+X)^{-1}\left(I-X A^{*}\right)$;

2. the inverse matrix of $A^{*}+c_{A}(X)$ is $(1 / 2)(A+X)$;

3. if $X \in \Omega(A)$ then $c_{A}(X) \in \Omega\left(A^{*}\right)$;

4. $c_{A}$ is a diffeomorphism, with $c_{A}^{-1}=c_{A^{*}}$.

Proof (1) It suffices to verify that $(A+X)\left(I-A^{*} X\right)=\left(I-X A^{*}\right)(A+X)$, which is immediate because $A A^{*}=\mathrm{id}$. For (2) we compute

$\left(A^{*}+\left(I-A^{*} X\right)(A+X)^{-1}\right)(1 / 2)(A+X)=(1 / 2)\left(A^{*} A+A^{*} X+I-A^{*} X\right)=I$.

Part (3) comes immediately from (2). Finally, by using (2) we obtain that

$$
\begin{aligned}
\left(c_{A^{*}} \circ c_{A}\right)(X) & = \\
\left(I-A c_{A}(X)\right)(1 / 2)(A+X) & = \\
\left(I-A\left(I-A^{*} X\right)(A+X)^{-1}\right)(1 / 2)(A+X) & = \\
(1 / 2)\left((A+X)-A+A A^{*} X\right) & =X .
\end{aligned}
$$

We shall need the following interesting properties, which are easy to prove:

Proposition 2 Let $X \in \Omega(A)$. Then

1. $X^{*} \in \Omega\left(A^{*}\right)$ and $c_{A^{*}}\left(X^{*}\right)=c_{A}(X)^{*}$;

2. $U X U^{*} \in \Omega\left(U A U^{*}\right)$ for any matrix $U \in O(n, \mathbb{K})$ and

$$
c_{U A U^{*}}\left(U X U^{*}\right)=U c_{A}(X) U^{*} ;
$$

3. if the matrix $X$ is invertible then $X^{-1} \in \Omega\left(A^{*}\right)$ and

$$
c_{A^{*}}\left(X^{-1}\right)=-A c_{A}(X) A .
$$




\subsection{Categorical open sets}

The results in this paragraph show that the domain of the Cayley transform in an orthogonal group is contractible.

The Lie algebra of $G=O(n, \mathbb{K})$ is formed by the skew-symmetric (resp. skew-hermitian) matrices,

$$
\mathfrak{g}=\mathfrak{o}(n, \mathbb{K})=\left\{X \in \mathcal{M}(n, \mathbb{K}): X+X^{*}=0\right\} .
$$

As a vector space $\mathfrak{g}=T_{I} G$, so the tangent space at any other point $A \in G$ is

$$
T_{A} G=L_{A}\left(T_{I} G\right)=\left\{Y \in \mathcal{M}(n, \mathbb{K}): A^{*} Y+Y^{*} A=0\right\} .
$$

Proposition 3 Let $X \in \mathfrak{o}(n, \mathbb{K})$ be a skew-symmetric (resp. skew-hermitian) matrix. Then $X$ has not real eigenvalues different from zero.

Proof Suppose that there exists $t \in \mathbb{R}$ such that $X v=v t$ for some $v \in \mathbb{K}^{n}$, $v \neq 0$. Then $v^{*} X v=v^{*} v t=|v|^{2} t$ is a real number and, consequently,

$$
v^{*} X v=\left(v^{*} X v\right)^{*}=v^{*} X^{*} v=v^{*}(-X) v=-v^{*} X v .
$$

Therefore $v^{*} X v$ is null. i.e. $|v|^{2} t=0$ hence $t=0$.

Corollary 1 The real vector space $T_{A} G$ of the matrices $Y$ such that $A^{*} Y+$ $Y^{*} A=0$ is contained in $\Omega(A)=\{Y \in \mathcal{M}(n, \mathbb{K}): A+Y$ is invertible $\}$.

Proof If $A+Y$ is not invertible then there exists $v \neq 0$ such that $Y v=-A v$ so $A^{*} Y v=-v$. This means that the skew-symmetric matrix $A^{*} Y$ has -1 as an eigenvalue, contradicting Proposition 3 , G.

Let $G=O(n, \mathbb{K})$. We shall denote by $\Omega_{G}(A)$ the open subset $\Omega(A) \cap G \subset$

Theorem 1 The generalized Cayley transform $c_{A}$ maps diffeomorphically $\Omega_{G}(A)$ onto $T_{A^{*}} G$, with $c_{A}(A)=0$. As a consequence, the open set $\Omega_{G}(A)$ is contractible.

The proof is an immediate consequence of Propositions 1, 2, 3 and Corollary 1

\section{Bott-Morse functions on Lie groups}

There is a deep relationship between the Cayley transform and Morse theory in Lie groups.

In this Section 3 we prove (Proposition 4) that one can integrate the gradient flow of any height function $h_{X}: G \rightarrow \mathbb{R}$ by applying the Cayley transform $c_{A^{*}}$ to a simple curve in $T_{A^{*}} G$, provided that $c_{A^{*}}(0)=A$ is a critical point. As a consequence we give a local model for the set $\Sigma$ of critical points of $h_{X}$ (Theorem 3). 


\subsection{Critical points of a height function}

Let $G=O(n, \mathbb{K})$ be an orthogonal group embedded in the euclidean space $E=\mathcal{M}(n, \mathbb{K})$. The euclidean metric is given by $\langle A, B\rangle=\Re \operatorname{Tr}\left(A^{*} B\right)$, the real part of the trace. I. A. Dynnikov and A. P. Veselov [21] and Volchenko and Kozachko [22] studied the height functions on $G$ (height with respect to some hyperplane), while $\mathrm{H}$. Duan [5] studied the distance functions on $G$ (distance to a given point). Up to a constant, both classes of functions are given by the formula

$$
h_{X}(A)=\Re \operatorname{Tr}(X A),
$$

for some matrix $X \in E$, as it is easy to prove.

Example 1 When $X=\operatorname{diag}\left(\lambda_{1}, \ldots, \lambda_{n}\right)$ is a positive real diagonal matrix, with $\lambda_{1}<\cdots<\lambda_{n}$, the function $h_{X}$ is a perfect Morse function, whose critical points are the diagonal matrices

$$
\operatorname{diag}\left(\varepsilon_{1}, \ldots, \varepsilon_{n}\right), \quad \epsilon_{k}= \pm 1 .
$$

This result is proven in [5] and [21], see also [16].

Example 2 On the other hand, the case $X=t I, t \in \mathbb{R}$, was first studied by T. Frankel in [8]. This time, the height map is a Bott-Morse function, invariant by the adjoint action. The set $\Sigma(n)$ of critical points is formed by the matrices $A$ such that $A^{2}=I$.

The two preceding examples are particular cases of the next Theorem, that we suppose is more or less folk. It gives a general description of the set of critical points of an arbitrary height function $h_{X}$.

A direct computation shows that the gradient of $h_{X}$ on $G$ is given by

$$
\left(\operatorname{grad} h_{X}\right)_{A}=\frac{1}{2}\left(X^{*}-A X A\right) .
$$

Moreover, if $A \in G$ is a critical point, then the Hessian operator $\left(H h_{x}\right)_{A}: T_{A} G \rightarrow$ $T_{A} G$ is given by

$$
\left(H h_{X}\right)_{A}(U)=-\frac{1}{2}(A X U+U X A), \quad U \in T_{A} G .
$$

We keep the notation of Example 2 ,

Theorem 2 Let $X \in E=\mathcal{M}(n, \mathbb{K})$ be an arbitrary matrix. Let $n_{0}$ be the dimension of its kernel and let $0<t_{1}<\ldots<t_{k}$ be the non-null (real) eigenvalues of $X X^{*}$, with multiplicities $n_{1}, \ldots, n_{k}$, that is $n_{0}+n_{1}+\cdots+n_{k}=$ $n$. Then the set of critical points of the height function $h_{X}$ is

$$
\Sigma\left(h_{X}\right) \cong O\left(n_{0}, \mathbb{K}\right) \times \Sigma\left(n_{1}\right) \times \cdots \times \Sigma\left(n_{k}\right) \quad \text { (diffeomorphism) }
$$


Proof First, if $Y=U D U^{*}$, then $\Sigma\left(h_{Y}\right)=U \Sigma\left(h_{D}\right) U^{*}$. On the other hand, if $X=U S$ is a polar decomposition ( $U$ orthogonal, $S$ hermitian) then $\Sigma\left(h_{X}\right)=\Sigma\left(h_{S}\right) U^{*}$. Finally, if $C=\operatorname{diag}\left(-I_{p},+I_{q}\right)$ then $\Sigma\left(h_{Y}\right)=C \Sigma\left(h_{Y C}\right)$. These properties prove that we can restrict ourselves to the case where $X$ is given by diagonal blocks

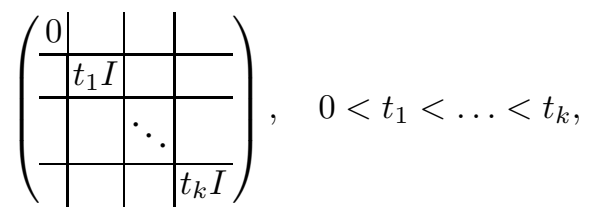

of size $n_{0}, n_{1}, \ldots, n_{k}$. The rest of the proof is a direct computation.

The transformations in the proof of Theorem 2 preserve the non-degenerate critical points, so we have the following corollary which completely characterizes the height functions which are Morse functions.

Corollary 2 The height function $h_{X}$ is Morse if and only if the matrix $X X^{*}$ is invertible and has $n$ different eigenvalues.

\subsection{Gradient flow and local model of the critical point set}

Our next Proposition is a generalization of the same result for the classical Cayley transform $c_{I}$ by Volchenko and Kozachko [22]. Following the terminology of these authors we shall call linearization the process of transforming the gradient flow of $h_{X}$ in $G$ to a flow in the Lie algebra.

Proposition 4 Let $h_{X}$ be an arbitrary height function on $G=O(n, \mathbb{K})$ and let $A$ be a critical point. The solution to the gradient equation

$$
\alpha^{\prime}=\frac{1}{2}\left(X^{*}-\alpha X \alpha\right)
$$

passing through $\alpha(0) \in \Omega_{G}(A)$ is the image by the generalized Cayley transform $c_{A^{*}}$ of the curve in $T_{A^{*}} G$ defined as

$$
\beta(t)=\exp (-X A t / 2) \cdot \beta_{0} \cdot \exp (-A X t / 2), \quad \beta_{0}=c_{A}(\alpha(0)) .
$$

Proof Notice that the matrices $X A$ and $A X$ are symmetric (hermitian) because $X^{*}=A X A$ and $A^{*} A=$ id.

First, from $A e^{X A}=e^{A X} A$ it follows that $A \beta+(A \beta)^{*}=0$, that is $\beta \in$ $T_{A^{*}} G$.

Now, from the definition of $\beta$, it is

$$
\beta^{\prime}=(-1 / 2)(X A \beta+\beta A X) .
$$

Let

$$
\alpha=c_{A^{*}} \circ \beta=(I-A \beta)\left(A^{*}+\beta\right)^{-1},
$$


hence

$$
\alpha\left(A^{*}+\beta\right)=I-A \beta .
$$

Derivation gives

$$
\alpha^{\prime}\left(A^{*}+\beta\right)+\alpha \beta^{\prime}=-A \beta^{\prime}
$$

that is

$$
\alpha^{\prime}\left(A^{*}+\beta\right)=-(A+\alpha) \beta^{\prime} .
$$

By Proposition 1, the inverse of $A^{*}+\beta=A^{*}+c_{A}(\alpha)$ is $(1 / 2)(A+\alpha)$, so

$$
\begin{array}{r}
\alpha^{\prime}= \\
(-1 / 2)(A+\alpha) \beta^{\prime}(A+\alpha)= \\
(+1 / 4)(A+\alpha)(X A \beta+\beta A X)(A+\alpha) .
\end{array}
$$

Now

$$
\beta=c_{A}(\alpha)=\left(I-A^{*} \alpha\right)(A+\alpha)^{-1}=(A+\alpha)^{-1}\left(I-\alpha A^{*}\right)
$$

implies that

$$
\beta(A+\alpha)=I-A^{*} \alpha
$$

and

$$
(A+\alpha) \beta=I-\alpha A^{*}
$$

so from Equation (2)

$$
\begin{array}{r}
4 \alpha^{\prime}= \\
(A+\alpha) X A\left(I-A^{*} \alpha\right)+\left(I-\alpha A^{*}\right) A X(A+\alpha)= \\
2 A X A-2 \alpha X \alpha= \\
2\left(X^{*}-\alpha X \alpha\right) .
\end{array}
$$

Remark 2 Indeed, when $X=A=I, \beta(t)=\exp (-t) \beta_{0}$ is the radial contraction to $\beta_{0}$.

We now show how the Cayley transform serves to give a local chart for the set of critical points. This result is completely new.

Let $h_{X}(A)=\Re \operatorname{Tr}(X A)$ be an arbitrary height function on the Lie group $G=O(n, \mathbb{K})$. Let $\Sigma$ be the set of critical points of $h_{X}$. If $A \in \Sigma$ is a critical point we denote by $S(A)$ the real vector space

$$
S(A)=\left\{\beta_{0} \in T_{A^{*}} G: X A \beta_{0}+\beta_{0} A X=0\right\} .
$$

Theorem 3 The Cayley map

$$
c_{A^{*}}: S(A) \rightarrow \Sigma \cap \Omega_{G}(A)
$$

is a diffeomorphism. 
Proof We need to prove that the curve $\beta(t)$ in Proposition 4 is constant if and only if $\beta_{0} \in S(A)$. This can be achieved by using Equation (1). Then, by Proposition 4, $c_{A^{*}}\left(\beta_{0}\right)$ is a critical point if and only if $\beta^{\prime}(t)=0$ for all $t$, if and only if

$$
X A \beta_{0}+\beta_{0} A X=0 .
$$

Example 3 Suppose $X=I$ and $\mathbb{K}=\mathbb{C}$. Then the critical points of $h_{I}$ are the matrices $A \in U(n)$ such that $A^{2}=I$. Such a matrix $A=A^{*}$ can be diagonalized to $D=\operatorname{diag}\left(\varepsilon_{1}, \ldots, \varepsilon_{n}\right), \varepsilon_{k}= \pm 1$. On the other hand, $\beta_{0} \in T_{A} G$ iff $A \beta_{0}$ is skew-symmetric, $A \beta_{0}=-\beta_{0}^{*} A$, while $\beta_{0} \in S(A)$ iff $A \beta_{0}+\beta_{0} A=0$. It follows that $\beta_{0}=\beta_{0}^{*}$.

So, for instance, the identity $I$ and its opposite $-I$ are critical points that are isolated because $S( \pm I)=0$. On the other hand, let $A=\operatorname{diag}\left(I_{p},-I_{q}\right)$. Then $\beta_{0} \in T_{A} G$ must be of the form

$$
\beta_{0}=\left(\begin{array}{cc}
0 & V^{*} \\
V & 0
\end{array}\right),
$$

which implies $\operatorname{dim} S(A)=2 p q$. This is in fact the dimension of the (critical) orbit of $A$, which is diffeomorphic to the Grasmannian $U(p+q) /(U(p) \times$ $U(q))$.

Example 4 Let $X=\operatorname{diag}\left(q_{1}, \ldots, q_{n}\right)$ be a diagonal matrix, with $q_{k} \neq 0$. Assume that $\left|q_{1}\right|<\ldots<\left|q_{n}\right|$. This time the gradient condition $A^{*} X^{*}=X A$ implies that a critical point has the form

$$
A=\operatorname{diag}\left( \pm\left|q_{1}\right| / q_{1}, \ldots, \pm\left|q_{n}\right| / q_{n}\right) .
$$

Since

$$
X A=A X=\operatorname{diag}\left(\varepsilon_{1}\left|q_{1}\right|, \ldots, \varepsilon_{n}\left|q_{n}\right|\right), \quad \varepsilon_{k}= \pm 1,
$$

it follows that $S(A)=0$. So all critical points are isolated.

\section{Applications to LS category}

4.1 The unitary group $U(n)$

W. Singhof proved in [19] that the LS category of the special unitary group $S U(n)$ is $n-1$, hence that of $U(n) \cong S^{1} \times S U(n)$ (diffeomorphism) is $n$. Although he obtained an explicit categorical covering by using the exponential map, his method has the inconvenience that a logarithm branch has to be chosen, hence introducing some technical complexities.

With the Cayley transform we obtain at each point $A \in G=U(n)$ a contractible open set $\Omega_{G}(A)$ diffeomorphic to the Lie algebra $\mathfrak{u}(n)$ (see Theorem 1). It is then very easy to find an explicit categorical covering of the group by $n+1$ open sets. 
Theorem 4 cat $U(n)=n$.

Proof Let $X \in U(n)$ be a unitary matrix and let $z \cdot$ id $\in U(n)$ be the diagonal matrix $\operatorname{diag}(z, \ldots, z)$, where $z \in \mathbb{C}$ is any complex number with $|z|=1$. Remember that $X \in \Omega(z \cdot$ id) iff the matrix $z \cdot$ id $+X$ is invertible. Let $\lambda_{1}, \ldots, \lambda_{n}$ be the eigenvalues of $X$, then after diagonalizing we obtain:

$$
z \cdot \mathrm{id}+X=U \operatorname{diag}\left(z+\lambda_{1}, \ldots, z+\lambda_{n}\right) U^{*}, \quad U \in U(n),
$$

meaning that $X \in \Omega\left(z \cdot\right.$ id) iff $\lambda_{i} \neq-z, \forall i=1, \ldots, n$.

Let us take $n+1$ different complex numbers $z_{0}, \ldots, z_{n}$, with $\left|z_{k}\right|=1$. Let $A_{k}=-z_{k}$.id. Since any matrix $X \in U(n)$ has at most $n$ different eigenvalues there is always some $-z_{k}$ which is not an eigenvalue of $X$, that is $X \in \Omega\left(A_{k}\right)$. This proves that $U(n)=\cup_{k=0}^{n} \Omega_{G}\left(A_{k}\right)$, hence cat $U(n) \leq$ $n$. On the other hand, as it is well known [11], a lower bound for the LS category is given by the length of the cup product. The cohomology of the unitary group being

$$
H(U(n))=\Lambda\left(x_{1}, x_{3}, \ldots, x_{2 n-1}\right)
$$

(see [4, p. 273]), the longest non-null cup product is $x_{1} \wedge x_{3} \wedge \cdots \wedge x_{2 n-1} \in$ $H^{n^{2}}$, hence $n=$ l.c.p. $\leq$ cat $U(n)$. Equality follows.

4.2 The symmetric spaces $U(2 n) / S p(n)$ and $U(n) / O(n)$

Theorem 5 cat $U(2 n) / S p(n)=n$.

The following proof is also a simplification of the original one [14].

Proof Following Mimura and Sugata, we consider the action $U \cdot X=$ $U X U^{T}$ of $U(2 n)$ on the manifold

$$
M=\left\{X \in U(2 n): X+X^{T}=0\right\} .
$$

This action turns out to be transitive with isotropy $S p(n)$. Then $U(2 n) / S p(n) \cong$ $M$. Here the symplectic group is identified (via complexification) with the subgroup of matrices $U \in U(2 n)$ such that $U J U^{T}=J$, where

$$
J=\left(\begin{array}{cc}
0 & -I_{n} \\
I_{n} & 0
\end{array}\right)
$$

Now, consider the manifold

$$
M^{\prime}=J M=\left\{Y \in U(2 n): Y^{T}=-J Y J\right\}
$$

which is diffeomorphic to $M$ because $J^{2}=-I$. 
Let $Y \in M^{\prime}$, let $\lambda \in \mathbb{C}$ with $|\lambda|=1$. Then $Y \in \Omega(\lambda I)$ if and only if $-\lambda$ is an eigenvalue of $Y$. But as remarked in [14], $Y v=-v \lambda$ implies $Y(J \bar{v})=-(J \bar{v}) \lambda$, which means that $Y$ can be diagonalized as

$$
Y=U\left(\begin{array}{cc}
D & 0 \\
0 & D
\end{array}\right) U^{*}, \quad D=\operatorname{diag}\left(\lambda_{1}, \ldots, \lambda_{n}\right),
$$

so

$$
Y+\lambda I=U\left(\begin{array}{cc}
D+\lambda I & 0 \\
0 & D+\lambda I
\end{array}\right) U^{*}
$$

showing that the maximum number of different eigenvalues of $Y$ is $n$. This implies that when taking $n+1$ different complex numbers $z_{0}, \ldots, z_{n}$, with $\left|z_{k}\right|=1$, the open sets $\Omega\left(-z_{k} I\right)$ will cover $M^{\prime}$.

It only remains to show that the Cayley contraction remains inside $M^{\prime}$. More explicitly, for any $z \in \mathbb{C},|z|=1$, let the Cayley map be

$$
c_{z I}: \Omega(z I) \rightarrow T_{\bar{z} I} G,
$$

let $Y \in \Omega(z I) \cap M^{\prime}$ and take the radial contraction $t c_{z I}(Y), t \in[0,1]$. In the same way of Proposition 2 it is easy to prove that

$$
c_{z I}\left(Y^{T}\right)=-c_{z I}(Y)^{T}
$$

and that

$$
c_{z J}(-J Y J)=-J c_{z I}(Y) J .
$$

This implies that the image $c_{\bar{z} I}\left(t c_{z I}(Y)\right.$ of the contraction by the inverse Cayley map is contained in $M^{\prime}$.

Hence cat $U(2 n) / S p(n) \leq n$. On the other hand ([15, p. 149]),

$$
H(U(2 n) / S p(n))=\Lambda\left(x_{1}, x_{5}, x_{9}, \ldots, x_{4 n-3}\right)
$$

so $n=$ l.c.p. $\leq$ cat $U(2 n) / S p(n)$. Equality follows.

Theorem 6 cat $U(n) / O(n)=n$.

The proof is completely analogous to the preceding one.

\subsection{The symplectic group $S p(2)$}

The LS category of $G=S p(2)$ was computed for the first time by P. Schweitzer [18], who proved that cat $S p(2)=3$.

By using the Morse theory explained above we shall easily obtain an explicit covering by four categorical open sets.

Let us consider the four critical points of a height function $h_{X}$ as in Example 1 of Section 3, namely: the identity $I=\operatorname{diag}(1,1), P=\operatorname{diag}(-1,1)$, $-P$ and $-I$.

Theorem $7\left\{ \pm \Omega_{G}(I), \pm \Omega_{G}(P)\right\}$ is a categorical covering of $G=S p(2)$. 
Proof Remember that $X \in \Omega_{G}(A)$ means that $A+X$ is invertible. First we see that the open sets $\Omega_{G}(I)$ and $\Omega_{G}(-I)$ cover the whole group excepting the orbit $U P U^{*}$ of the matrix $P$ by the adjoint action. In fact, by diagonalization, this orbit is formed by the matrices having 1 and -1 as eigenvalues and is diffeomorphic to the sphere $S^{4}$, so it can be covered by stereographic projection.

Explicitly, we must prove that given $X=\left(x_{i j}\right)=U P U^{*}$ in the orbit of $P$, either $P+X$ or $-P+X$ is invertible.

Since $P^{2}=X^{2}=I$ we have

$$
(P+X)^{2}=2 I+P X+X P=2 \operatorname{diag}\left(1-x_{11}, 1+x_{22}\right) .
$$

Then $P+X$ is invertible if and only if $x_{11} \neq 1$ and $x_{22} \neq-1$. Suppose $x_{11}=1$. The condition $X^{*} X=I$ means that the columns of $X$ form an orthonormal basis of $\mathbb{H}^{2}$ for the hermitian product $\langle v, w\rangle=v^{*} w$. Then, $x_{11}=1$ implies $x_{12}=x_{21}=0$. But since $X$ is in the orbit of $P$, it must be $x_{22}=-1$. Hence $X=-P$. The same conclusion is obtained from $x_{22}=$ -1 . So in fact $\Omega_{G}(P)$ covers all the orbit of $P$, excepting $-P$. Since $-P \in$ $\Omega_{G}(-P)$, the proof is done.

Remark 3 The cohomology of the symplectic group is [15, p. 119]

$$
H(S p(n))=\Lambda\left(x_{3}, x_{7}, \ldots, x_{4 n-1}\right)
$$

so the longest non-null product is $x_{3} \wedge x_{7} \wedge \cdots \wedge x_{4 n-1}$ and l.c.p. $S p(n)=n$. However Schweitzer [18] was able to prove that cat $S p(n) \geq n+1$ for $n \geq 2$.

\subsection{Left eigenvalues of symplectic matrices}

Obviously it is not worthy to apply the method of critical points above to

the symplectic group $S p(n), n>2$ (for instance it is known that cat $S p(3)=5$ [7]). Instead, in this section we discuss the possibility of extending the eigenvalue method of paragraphs 4.1 and 4.2 to the symplectic setting.

We shall briefly explain the underlying difficulties. First, it is necessary to endow the quaternionic space $\mathbb{H}^{n}$ with the structure of a right $\mathbb{H}$-vector space, in order to obtain the usual results for the matrix associated to a linear map. Second, the theory of right eigenvalues is well established, including diagonalization of symplectic matrices [1, 2, 6, 24]. However, for a matrix $A \in S p(n)$ and a quaternion $\sigma \in \mathbb{H}$, the condition that $A-\sigma I$ be invertible is not related to $\sigma$ being a right eigenvalue. Instead we must consider left eigenvalues.

Definition 2 A quaternion $\sigma \in \mathbb{H}$ is a left eigenvalue of the matrix $A \in$ $\mathcal{M}(n, \mathbb{H})$ if and only if there exists $v \in \mathbb{H}^{n}, v \neq 0$, such that $A v=\sigma v$. 
Unfortunately, very little is known about left eigenvalues of quaternionic matrices. Their existence, number, and methods for computing them are only partially understood, see Zhang's paper [25] for a recent review. For $n=2$ two of the authors were able to prove the following Theorem, based on previous results by Huang and So [9].

Theorem 8 ([12]) A symplectic matrix $A \in S p(2)$ has either one, two or infinite left eigenvalues. The latter case can only occur when

$$
A=L_{q} \circ R_{\theta}=\left(\begin{array}{cc}
q \cos \theta & -q \sin \theta \\
q \sin \theta & q \cos \theta
\end{array}\right), \quad q \in \mathbb{H},|q|=1, \theta \in \mathbb{R}, \sin \theta \neq 0 .
$$

As a corollary it is possible to obtain the following deceptive result.

Corollary 3 ([13]) Let $\sigma_{0}, \ldots, \sigma_{3}$ be four arbitrary quaternions of module 1. Then the categorical open sets $\Omega_{G}\left(\sigma_{0} \cdot I\right), \ldots, \Omega_{G}\left(\sigma_{3} \cdot I\right)$ do not cover the group $G=S p(2)$.

Acknowledgements We acknowledge many useful discussions with D. Tanré.

\section{References}

1. Baker, A. Right eigenvalues for quaternionic matrices: A topological approach. Linear Algebra Appl. 286, No.1-3, 303-309 (1999)

2. Brenner, J. L. Matrices of quaternions. Pac. J. Math. 1, 329-335 (1951).

3. Cayley, A. Sur quelques propriétés des déterminants gauches. J. Reine Angew. Math. 32, 119-123 (1846).

4. Cornea, O.; Lupton, G.; Oprea, J.; Tanré, D. Lusternik-Schnirelmann category. Mathematical Surveys and Monographs 103. American Mathematical Society (2003).

5. Duan, H. Morse functions and Cohomology of Homogeneous Spaces, in Pragacz, Piotr (ed.), Topics in cohomological studies of algebraic varieties. Impanga lecture notes. Trends in Mathematics. Birkhäuser, 105-133 (2005).

6. Farenick, D. R.; Pidkowich, B. A. F. The spectral theorem in quaternions. Lin. Alg. Appl. 371, 75-102 (2003).

7. Fernández-Suárez, L.; Gómez-Tato, A.; Strom, J.; Tanré, D. The LyusternikSchnirelmann category of $S p(3)$. Proc. Am. Math. Soc. 132, No. 2, 587-595 (2004).

8. Frankel, T. Critical submanifolds of the classical groups and Stiefel manifolds. Differ and Combinat. Topology, Sympos. Marston Morse, Princeton, 37-53 (1963).

9. Huang, L.; So, W. On left eigenvalues of a quaternionic matrix. Linear Algebra Appl. 323 , No.1-3, 105-116 (2001).

10. Iwase, N.; Mimura, M. L-S categories of simply-connected compact simple Lie groups of low rank. Arone, Gregory (ed.) et al., Categorical decomposition techniques in algebraic topology. Proceedings of the international conference in algebraic topology, Isle of Skye, UK, June 2001. BirkhŁuser. Prog. Math. 215, 199-212 (2004).

11. James, I.M. Lusternik-Schnirelmann category. James, I. M. (ed.), Handbook of algebraic topology. North-Holland, 1293-1310 (1995).

12. Macías-Virgós, E.; Pereira-Sáez, M. J. Left eigenvalues of $2 \times 2$ symplectic matrices. Electron. J. Linear Algebra Vol. 18, 274-280 (2009).

13. Macías-Virgós, E.; Pereira-Sáez, M. J. Symplectic matrices with prescribed eigenvalues. preprint 2009. 
14. Mimura, M.; Sugata, K. On the Lusternik-Schnirelmann category of symmetric spaces of classical type. Iwase, N. (ed.) et al., Proceedings of the conference on groups, homotopy and configuration spaces, University of Tokyo, Japan, July 5-11, 2005 in honor of the 60th birthday of Fred Cohen. Coventry: Geometry \& Topology Publications. Geometry and Topology Monographs 13, 323-334 (2008).

15. Mimura, M.; Toda, H. Topology of Lie groups, I and II. Translations of Mathematical Monographs 91. American Mathematical Society (1991).

16. Pereira-Sáez, M. J. Aplicación traza, transformación de Cayley y categoría LS de los grupos de Lie clásicos. Publ. Dpto. Xeom. e Top. 113. Universidade de Santiago de Compostela (2008).

17. Postnikov, M. Lie groups and Lie algebras. Moscow, Lectures in Geometry 5 (1994).

18. Schweitzer, P. Secondary cohomology operations induced by the diagonal mapping. Topology 3, 337-355 (1965).

19. Singhof, W. On the Lusternik-Schnirelmann Category of Lie Groups I. Math. Z. 145, 11-116 (1975).

20. Singhof, W. On the Lusternik-Schnirelmann Category of Lie Groups II. Math. Z. 151, 143-148 (1976).

21. Veselov, A. P.; Dynnikov, I. A. Integrable gradient flows and Morse theory. St. Petersbg. Math. J. 8, No. 3, 429-446 (1997); translation from Algebra Anal. 8, No. 3, 78-103 (1996).

22. Volchenko, K. Y.; Kozachko, A. N. Integrable gradient flows on classic manifolds and Morse theory. Mosc. Univ. Math. Bull. 52, No.3, 9-15 (1997); translation from Vestn. Mosk. Univ., Ser. I 1997, No.3, 9-15 (1997).

23. Weyl, H. The classical groups, their invariants and representations. Princeton Landmarks in Mathematics. Princeton University Press (1997).

24. Zhang, F. Quaternions and matrices of quaternions. Linear Algebra Appl. 251, 21-57 (1997).

25. Zhang, F. Geršgorin type theorems for quaternionic matrices. Linear Algebra Appl. 424, No. 1, 139-153 (2007). 